\title{
A uniform spatial allocation strategy in solving water resources optimization dispatch problem
}

\author{
Jiahui Sun ${ }^{1,2}$, Xiaohui Lei ${ }^{2 *}$, Ji Liang ${ }^{1}$, Chao Wang ${ }^{2}$, Liang Men ${ }^{1}$ \\ ${ }^{1}$ School of Hydropower and Information Engineering, Huazhong University of Science and Technology, Wuhan 430074, China \\ ${ }^{2}$ State Key Laboratory of Simulation and Regulation of Water Cycle in River Basin, China Institute of Water Resources and Hydropower \\ Research,1 Fuxing Beijing 100038, China
}

\begin{abstract}
Along with social economy development, the total water consumption increased year by year. The conflicts between water supply and water consumption is growing. Water resources optimization dispatch, which is used to allocate water resources to meet the demand of water user on both time scale and spatial scale, plays an important role in water resources management. Aiming at improving the spatial allocation ability of traditional optimization algorithms, a uniform spatial allocation strategy is proposed accordingly. The proposed method is used to improve the performance of optimization algorithm to obtain solutions which can uniform the water supply in spatial scale. Simulation results show that the maximum ratio of water deficiency gained by the proposed method is smaller than that obtained by the original algorithm. The proposed method is effective to balance the demand of water users in spatial scale.

Copyright (C) 2018 Elsevier Ltd. All rights reserved.

Selection and peer-review under responsibility of the scientific committee of the $10^{\text {th }}$ International Conference on Applied Energy (ICAE2018).
\end{abstract}

\section{Introduction}

Along with social economy development, the total water consumption increased year by year. The conflicts between water supply and water consumption is growing. Water resources optimization dispatch, which is used to allocate water resources to meet the demand of water user on both time scale and spatial scale, plays an important role in water resources management ${ }^{[1-2]}$. In order to solve this problem, numerous methods have been proposed in recent years, linear programing (LP) ${ }^{[3,4]}$, none linear programming (NLP) ${ }^{[5,6]}$, dynamic programs (DP) ${ }^{[7,8]}$, discrete differential dynamic programming (DDDP) ${ }^{[9,10]}$, heuristic algorithms, such as particle swarm optimization (PSO) [11-13], genetic algorithm (GA) ${ }^{[14,15]}$, differential evolution (DE) ${ }^{[16,17]}$, cultural algorithm (CA) ${ }^{[18]}$, ant colony algorithm $(\mathrm{ACO})^{[19,20]}$, ant colony optimization for continuous domain $\left(\mathrm{ACO}_{\mathrm{R}}\right)^{[21]}$. These algorithms have been widely utilized in WROD. While all these algorithms try to optimal the water deficit on time scale by controlling the process of reservoir storage, lacking consideration the uniform spatial allocation demand of all the water users. In this paper, a uniform spatial allocation strategy has been proposed, which could efficiently improve the performance of optimization algorithm to obtain solutions which can uniform the water supply in spatial scale.

The reminder of this paper is organized as follows, Section 2 introduces the formulation of WORD problem as well as the problem solving strategy. In Section 3, the proposed method is applied to solve WORD in GuiJiang River, and the results are analysed. Finally, conclusions are summarized in Section 4.

\section{Methodology}

\subsection{Problem formulation}

\subsubsection{Objective function}

In order to reflect the water deficient problem in river basin, the sum of each water user's water supply deficient volume is chosen to be the objective.

$$
\text { deficient }=\sum_{i=1}^{\mathrm{n}} \sum_{t=1}^{T}\left(D_{i, t}-S_{i, t}\right)
$$

where $D_{i, t}$ is the demand water of the $i$-th water user at the $t$-th period, $S_{i, t}$ is the supply water of the $i$-th water user at the $t$-th period. $n$ is the number of water users in river basin, $T$ is the scheduling period.

\subsubsection{Constraints}

(1) Water balance constraint

$$
V_{t+1}=V_{t}+\left(I_{t}-Q_{t}\right) \Delta t
$$


$V_{t}$ is the capacity storage of the reservoir. $I_{t}$ is the average inflow in period t, $Q_{t}$ is the average outflow in period t, $\Delta t$ is the time span of a single period.

(2) Water level constraint

$$
Z_{t}^{\min } \leq Z_{t} \leq Z_{t}^{\max }
$$

$Z_{t}^{\min }$ and $Z_{t}^{\max }$ are the maximum and the minimum water level of the reservoir at the $t$-th period.

(3) Flow constraint

$$
Q_{t}^{\min } \leq Q_{t} \leq Q_{t}^{\max }
$$

$Q_{t}^{\max }$ is the maximum outflow reservoir in period t. $Q_{t}^{\min }$ is the minimum outflow.

(4) Section flow constraint

$$
\begin{gathered}
Q_{\mathrm{sec} t} \geq Q_{\mathrm{sec} t}^{\min } \\
Q_{\mathrm{sec} t}^{\min }
\end{gathered}
$$

$t$.

\subsection{River basin topology structure generalization}

The various physical entities to be considered in the water resources management problem include rivers, catchments, reservoirs, water users and water intakes. Among them, river is the link connecting the remaining entities. The river entity also contains sub-entities(key river sections). The key sections include control section, the inflow section of the catchment, the section of the reservoir, the water intake section, the first and last sections of the river and section at the intersection. The catchment is an entity formed by generalizing the inflow water in the basin, generally taking the upstream area of flow station on the main stream or reservoir as a catchment.

Establishing the generalized topological structure of the river basin is the basis for studying the water resources management problem. The model logical structure directly affects the running speed of the computer, and a reasonable logical structure is easy for computer's implement. The process of compiling the topology structure is as follows: Firstly, traverse all entities and store the river entities. and traverse the key sections of the current river and store them in the model calculate sequence by river. Next, during the traversal process, judge the type of current key section. (1)If the current section is control section or the first and last sections of the river, store the current section and jump to the next section; (2)If the current section is the inflow section of the catchment, the section of the reservoir or the water intake section, the corresponding catchment, reservoir and water intake is stored in the associated entity class of the current section, and then jumped to the next section; (3)If the current section is section at the intersection, whether the other river's topology has been compiled should be determined. If the topology has been compiled, store the current section and jump to the next section of the current river. Otherwise, the current node is skipped and store the first key section of the crossing river. Finally, until all the rivers' topology structures have been successfully compiled, end the process and the river basin model calculate sequence is finished.

\subsection{Differential evolution algorithm}

Differential evolution algorithm (DE algorithm) is a new type of algorithm. Its core principle is based on the theory of swarm intelligence. Because the DE algorithm has a very fast speed of convergence speed and a strong global search ability, it has been applied to solve many complex optimization problems in many fields since the theory was put forward.

The basic process of differential evolution algorithm consists of three parts: mutation, intersection and selection. The process of differential evolution algorithm is similar to the selection process of nature. It also complies with the survival competition criterion of "survival of the fittest". The realization of this criterion depends on the guidance of fitness value function. The fitness value is a quantitative measure that reflects the relative goodness of the target individual in the existing population. The fitness value is calculated by the fitness function, and the fitness function has different manifestations due to different specific scenarios.

In the DE algorithm, single population individuals are generated by floating point vector coding. The function of the DE algorithm is as follows, First, two different individuals are selected from the initial parent population to generate a difference vector between the two individuals; Second, another individual among the parent population is selected. and the selected individual is summed with the previously obtained difference vector to generate a new experimental individual; Then, crossoperates the initial parent population and the calculated experimental individual to generate a new individual; Finally, choose the better one between the initial parent individual, the newly generated individual and save the individual, which meets the target requirements.

\subsection{Uniform spatial allocation strategy}

The uniform spatial allocation strategy is based on the river basin calculation partition division method. The calculation partition division method divides the water basin according to the river basin model calculate sequence. The principle of partitioning river basin calculation partitions is to divide the calculate sequence into different partitions by sections at the intersection and water intake sections. Each partition only has one water intake section. Partition statistics and stores its upstream partitions which have hydraulically connection with current partition. The uniform spatial allocation strategy adjusts the spatial uneven water resource allocation problem on the basis of differential evolution algorithm. Details are shown as following,

Step 1: Statistics the amount of natural inflow and reservoir storage variation in each partition to partition available water supply;

Step 2: Statistics the remaining water of the upstream partitions hydraulically linked to current partition, and then balance the available water and demand water of the 
current partition. If the available water is sufficient, supply water is equal to demand water, else supply water is equal to available water (Need consider the minimum ecologic flow of river);

Step 3: According to the preliminary allocation result, calculate the water shortage rate of each partition. Then traverse the upstream partitions hydraulically linked to current partition (ignore partitions whose water shortage are higher than current partition). Statistics the water deficiencies of all relevant partitions and recalculate the overall shortage rate, all relevant partitions are supplied by the overall shortage rate.

Step 4: Allocate the balanced water shortage to each water intake for reduction, re-simulate and update the fitness.

\section{Case study}

\subsection{Description of case study}

GuiJiang river, one of the major tributaries of the XiJiang river system, originated from the first peak of GuangxiCang'er Mountain. GuiJiang river flows through LingChuan County, Guilin City, YangShuo County and PingLe County, merges with GongCheng River into GuiJiang River. Then it flows through ZhaoPing County,
CangWu County, and WuZhou City into the XiJiang River. It has a total length of 426 kilometers and a river basin area of 19,288 square kilometers. In this case, six reservoirs in GuiJiang River Basin are considered: Fuzikou Reservoir, Chuanjiang Reservoir, Xiaorongjiang Reservoir, Qingshitan Reservoir, Zhaoping Reservoir and Jingnan Reservoir. The water users in the river basin are generalized in the form of administrative district, including: Xing'an County, Lingchuan County, Guilin City, Yangshuo County, Pingle County and Zhangzhou City. The catchments are the upstream area of reservoirs and Guilin City. (shown in Fig.1). The problem of uneven distribution of water supply between upstream and downstream water users is very easy to occur in dry season. Therefore, it is imperative to use the uniform spatial allocation strategy.

The measured runoff data of each flow station in dry season of Guijiang River Basin is used as the water income input, and the water consumption reported by each administrative district is used as the water demand input. The water regulation optimization model is established according to the rules in problem formulation. According to the actual incoming water and consumption water, the water resources optimization dispatch is carried out under the condition under the condition of uniform spatial allocation strategy and no strategy respectively..

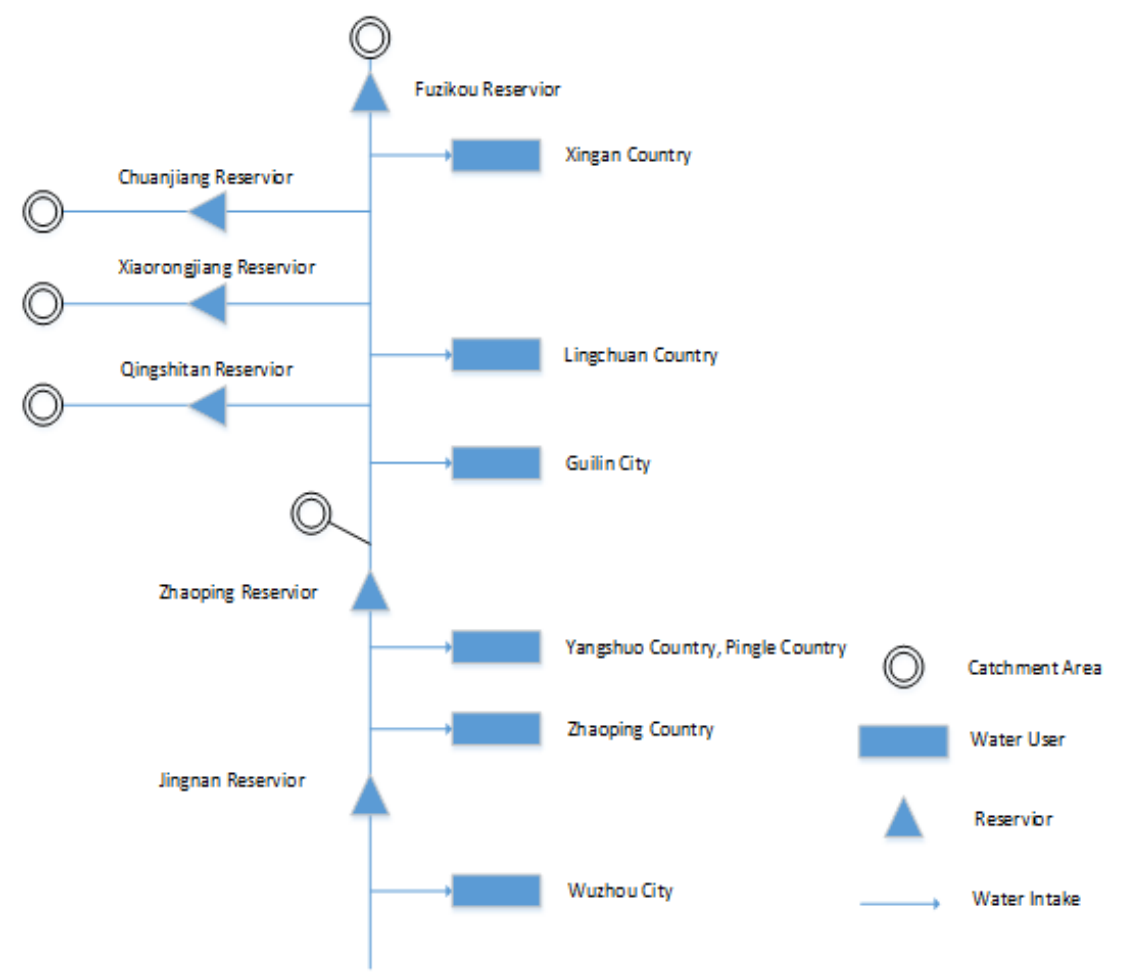

Fig.1 GuiJiang river basin topology network

\subsection{Results analysis}

In this section, case 1 is simulated with method mentioned in Section 2.2, case 2 is simulated with methods mentioned in Section 2.2 and 2.3. Simulation of this problem is based upon a Java implementation running on a personal computer, Winds7 Ultimate server Pack1 x64, Intel(R) Core(TM) i5-2320 CPU@ 3.00GHZ, RAM 4.00GB.
Detail results are shown as follows.

Fig. 2 shows the difference between each water user's deficient rate between case 1 and case 2 . In case 1 , upstream water user's assurance rate of water supply is much higher than downstream water users. In case 2, by using the uniform spatial allocation strategy, water supply assurance rate of water user upstream and downstream is basically uniformed. Meanwhile, the sum of each water user's water supply deficient volume in case 2 is a little 
lower than case 1.
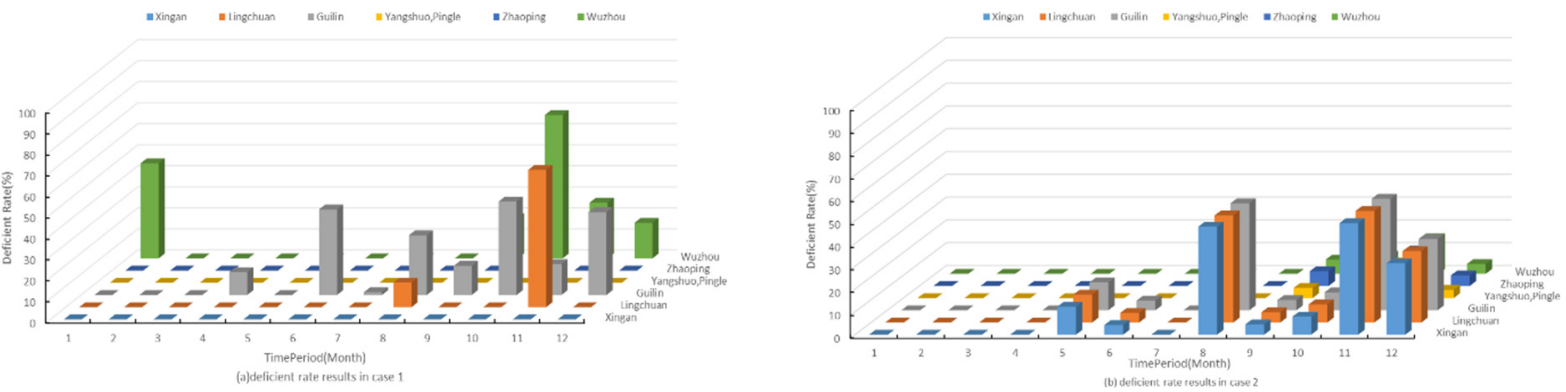

Fig.2. Comparison of deficient rate results in case 1 and case 2

Without using the uniform spatial allocation strategy, we can see that XingAn and Yangshuo Country in upstream has plentiful water all year, while GuiLin and Wuzhou City in downstream suffers seriously water shortage, shown in Table1. From Table 2, we can see

obviously that the water shortage is fairly shared between XingAn Country and Guilin City, which means that the uniform spatial allocation strategy can uniform the water supply in spatial scale.

Table 1: The water supply and demand results of XingAn and GuiLin in Case 1

\begin{tabular}{|c|c|c|c|c|c|c|}
\hline \multirow[b]{2}{*}{ Month } & \multicolumn{3}{|c|}{ XingAn } & \multicolumn{3}{|c|}{ GuiLin } \\
\hline & $\begin{array}{c}\text { Demand } \\
\text { Water }\left(10^{4} \mathrm{~m}^{3}\right)\end{array}$ & $\begin{array}{c}\text { Supply } \\
\text { Water }\left(10^{4} \mathrm{~m}^{3}\right) \\
\end{array}$ & $\begin{array}{l}\text { Deficient } \\
\text { Rate }(\%)\end{array}$ & $\begin{array}{c}\text { Demand } \\
\text { Water }\left(10^{4} \mathrm{~m}^{3}\right)\end{array}$ & $\begin{array}{c}\text { Supply } \\
\text { Water }\left(10^{4} \mathrm{~m}^{3}\right)\end{array}$ & $\begin{array}{c}\text { Deficient } \\
\text { Rate }(\%)\end{array}$ \\
\hline 1 & 1885.72 & 1885.72 & 0 & 7467.37 & 7467.37 & 0 \\
\hline 2 & 1954.59 & 1954.59 & 0 & 8022.23 & 8022.23 & 0 \\
\hline 3 & 1755.67 & 1755.67 & 0 & 21797.19 & 21797.19 & 0 \\
\hline 4 & 2263.98 & 2263.98 & 0 & 14638.78 & 13040.70 & 10.92 \\
\hline 5 & 2346.92 & 2346.92 & 0 & 15239.87 & 15239.87 & 0 \\
\hline 6 & 2382.31 & 2382.31 & 0 & 10756.16 & 6378.67 & 40.70 \\
\hline 7 & 2282.31 & 2282.31 & 0 & 10756.16 & 10610.61 & 1.35 \\
\hline 8 & 2215.79 & 2215.79 & 0 & 15794.73 & 11311.47 & 28.38 \\
\hline 9 & 1738.61 & 1738.61 & 0 & 22398.28 & 19294.63 & 13.86 \\
\hline 10 & 2163.98 & 2163.98 & 0 & 14638.78 & 8132.38 & 44.45 \\
\hline 11 & 1819.20 & 1819.2 & 0 & 13505.94 & 11518.72 & 14.71 \\
\hline 12 & 1729.22 & 1729.22 & 0 & 12881.72 & 7808.365 & 39.38 \\
\hline
\end{tabular}

Table 2: The water supply and demand results of XingAn and GuiLin in Case 2

\begin{tabular}{|c|c|c|c|c|c|c|}
\hline \multirow[b]{2}{*}{ Month } & \multicolumn{3}{|c|}{ XingAn } & \multicolumn{3}{|c|}{ GuiLin } \\
\hline & $\begin{array}{c}\text { Demand } \\
\text { Water }\left(10^{4} \mathrm{~m}^{3}\right) \\
\end{array}$ & $\begin{array}{c}\text { Supply } \\
\text { Water }\left(10^{4} \mathrm{~m}^{3}\right) \\
\end{array}$ & $\begin{array}{c}\text { Deficient } \\
\text { Rate }(\%)\end{array}$ & $\begin{array}{c}\text { Demand } \\
\text { Water }\left(10^{4} \mathrm{~m}^{3}\right)\end{array}$ & $\begin{array}{c}\text { Supply } \\
\text { Water }\left(10^{4} \mathrm{~m}^{3}\right) \\
\end{array}$ & $\begin{array}{l}\text { Deficient } \\
\text { Rate(\%) }\end{array}$ \\
\hline 1 & 1885.72 & 1885.72 & 0 & 7467.37 & 7467.37 & 0 \\
\hline 2 & 1954.59 & 1954.59 & 0 & 8022.23 & 8022.23 & 0 \\
\hline 3 & 1755.67 & 1755.67 & 0 & 21797.19 & 21797.19 & 0 \\
\hline 4 & 2263.98 & 2263.98 & 0 & 14638.78 & 14638.78 & 0 \\
\hline 5 & 2346.92 & 2059.30 & 12.25 & 15239.87 & 13372.17 & 12.25 \\
\hline 6 & 2382.31 & 2281.45 & 4.23 & 10756.16 & 10300.79 & 4.23 \\
\hline 7 & 2282.31 & 2282.31 & 0 & 10756.16 & 10756.16 & 0 \\
\hline 8 & 2215.79 & 1165.13 & 47.42 & 15794.73 & 8377.12 & 46.96 \\
\hline 9 & 1738.61 & 1660.04 & 4.52 & 22398.28 & 21386.02 & 4.52 \\
\hline 10 & 2163.98 & 1993.00 & 7.90 & 14638.78 & 13482.13 & 7.90 \\
\hline 11 & 1819.2 & 928.83 & 48.94 & 13505.94 & 6895.75 & 48.94 \\
\hline 12 & 1729.22 & 1187.44 & 31.33 & 12881.72 & 8845.79 & 31.33 \\
\hline
\end{tabular}

\section{Conclusion}

In this paper, a water resources optimization dispatch problem on both time scale and spatial scale is proposed. The sum of each water user's water supply deficient volume is used to be the criterion of water allocation in river basin. To solve this problem, a uniform spatial 
allocation strategy is proposed to handle the allocation unbalance on spatial scale. Simulation results demonstrate that single differential evolution algorithm cannot solve the unbalance problem on spatial scale without adding constraints. On the basis that calculating order is from upstream to downstream, the water supply of upstream water user is ensured preferentially. But with the combination of the DE algorithm and uniform spatial allocation strategy, the demand of water users on both time scale and spatial scale has been taken into consideration. Therefore, the uniform spatial allocation strategy can significantly improve the performance of optimization algorithm to obtain solutions which can uniform the water supply in spatial scale.

\section{References}

1. Bin M A, Xie J, Ben R, et al. Model of water resources dispatch and management based on distributed component technology. Journal of Hydraulic Engineering, 2000, 31(12):26-30.

2. Yang W, Yang C, Sun C. The application of penal function method in real time dispatch of water resources system. Journal of Heilongjiang Hydraulic Engineering College, 2002, 55(82-83):522-526.

3. J.S. Windsor. Optimization model for the operation of flood control systems. Water Resources Research. 9 (1973) 1219-26.

4. J.-H. Yoo. Maximization of hydropower generation through the application of a linear programming model. Journal of hydrology. 376 (2009) 182-7.

5. J.L.B. Brandão. Performance of the equivalent reservoir modelling technique for multi-reservoir hydropower systems. Water resources management. 24 (2010) 3101-14.

6. J. Catalão, H. Pousinho, V. Mendes. Scheduling of head-dependent cascaded hydro systems: Mixedinteger quadratic programming approach. Energy Conversion and Management. 51 (2010) 524-30.

7. R.R. Shoults, R.K. Chakravarty, R. Lowther. Quasistatic economic dispatch using dynamic programming with an improved zoom feature. Electric power systems research. 39 (1996) 215-22.

8. D.N. Kumar, F. Baliarsingh. Folded dynamic programming for optimal operation of multireservoir system. Water Resources Management. 17 (2003) 337-53.

9. V. Te Chow, G. Cortes-Rivera, A. No. Application of DDDP in water resources planning. University of Illinois at Urbana-Champaign, Water Resources Center1974.

10. M. Heidari, V.T. Chow, P.V. Kokotović, D.D. Meredith. Discrete differential dynamic programing approach to water resources systems optimization. Water Resources Research. 7 (1971) 273-82.

11. J. Chuanwen, E. Bompard. A self-adaptive chaotic particle swarm algorithm for short term hydroelectric system scheduling in deregulated environment. Energy Conversion and Management. 46 (2005)
2689-96.

12. C.-t. Cheng, S.-1. Liao, Z.-T. Tang, M.-y. Zhao, Comparison of particle swarm optimization and dynamic programming for large scale hydro unit load dispatch. Energy Conversion and Management. 50 (2009) 3007-14.

13. R. Zhang, J. Zhou, Y. Lu, H. Qin, H. Zhang. A PSObased bacterial chemotaxis algorithm and its application. Advances in Neural Networks-ISNN 2011. Springer2011. pp. 219-27.

14. R. Wardlaw, M. Sharif. Evaluation of genetic algorithms for optimal reservoir system operation. Journal of Water Resources Planning and Management. 125 (1999) 25-33.

15. C.-T. Cheng, W.-C. Wang, D.-M. Xu, K. Chau. Optimizing hydropower reservoir operation using hybrid genetic algorithm and chaos. Water Resources Management. 22 (2008) 895-909.

16. Y. Lu, J. Zhou, H. Qin, Y. Li, Y. Zhang. An adaptive hybrid differential evolution algorithm for dynamic economic dispatch with valve-point effects. Expert Systems with Applications. 37 (2010) 4842-9.

17. X. Yuan, B. Cao, B. Yang, Y. Yuan. Hydrothermal scheduling using chaotic hybrid differential evolution. Energy Conversion and Management. 49 (2008) 3627-33.

18. X. Yuan, Y. Yuan. Application of cultural algorithm to generation scheduling of hydrothermal systems. Energy Conversion and Management. 47 (2006) 2192-201.

19. J. Cai, X. Ma, L. Li, Y. Yang, H. Peng, X. Wang. Chaotic ant swarm optimization to economic dispatch Electric Power Systems Research. 77 (2007) 1373-80.

20. L. Mo, P. Lu, C. Wang, J. Zhou. Short-term hydro generation scheduling of Three Gorges-Gezhouba cascaded hydropower plants using hybrid MACSADE approach. Energy Conversion and Management. 76 (2013) 260-73.

21. A. Fetanat, G. Shafipour. Generation maintenance scheduling in power systems using ant colony optimization for continuous domains based 0-1 integer programming. Expert Systems with Applications. 38 (2011) 9729-35. 